\title{
Dinâmica Espaço-Temporal de COVID-19 nas Regionais Administrativas de Belo Horizonte, Minas Gerais - Brasil
}

\section{Spatial-Temporal Dynamic of Covid-19 in the Administrative Regions of Belo Horizonte, Minas Gerais - Brasil}

\author{
Úrsula de Azevedo Ruchkys \\ Professora Associada do Instituto de Geociências - UFMG, Brasil \\ Programa de Pós-Graduação em Análise e \\ Modelagem de Sistemas Ambientais e do Programa de \\ Pós-Graduação em Geografia. \\ ularuchkys@yahoo.com.br
}

Nathan Carlo Martins Graduando do Curso de Geologia do Instituto de Geociências - UFMG, Brasil nathan.carlo33@gmail.com

Eric Oliveira Pereira Instituto de Geociências Universidade Federal de Minas Gerais, Brasil ericpereiraufmg@gmail.com

Edimar Olegário de Campos Júnior Bolsista CAPES, Pós-Doutorando Instituto de Geociências Universidade Federal de Minas Gerais, Brasil edimarcampos@yahoo.com.br

Matheus Luiz Cortez Doutorando, Bolsista CAPES Instituto de Geociências Universidade Federal de Minas Gerais, Brasil cortez.agro@gmail.com

Sonia Maria Carvalho Ribeiro Professora do Instituto de Geociências - UFMG, Brasil

Programa de Pós-Graduação em Análise e Modelagem de Sistemas Ambientais, UFMG, Brasil sonicarvalhoribeiro@ufmg.br

\section{Resumo}

As epidemias são uma grande preocupação da espécie humana e os patógenos virais representam um perigo sempre presente para a saúde global, com destaque para evento recente da epidemia de COVID-19 causada pelo Coronavírus SARS-CoV-2. O artigo tem como objetivo analisar as taxas de mortalidade da COVID-19 e explorar suas associações com indicadores demográficos e sociais nas nove regiões administrativas do município de Belo Horizonte, capital de Minas Gerais, segundo mais populoso Estado do Brasil. O estudo considera e analisa os padrões espaço-temporais de óbitos por COVID-19 reportados nos boletins epidemiológicos da Prefeitura de Belo Horizonte (PBH) 
considerando o período da Semana Epidemiológica (SE), SE 23/2020 (31 de maio de 2020) até SE 21/2021 (29 de maio de 2021). Os resultados indicam que as regionais administrativas tiveram comportamento diferenciado em relação a COVID-19 e que esse desempenho, associa-se, predominantemente, às características demográficas e de vulnerabilidade social.

Palavras-chave: Epidemia; Taxas de Mortalidade; Indicadores Demográficos e Sociais

\begin{abstract}
Epidemics are a great concern to the human species and viral pathogens represent an ever-present danger to global health, as highlighted by the recent event of the COVID-19 pandemic caused by Coronavirus SARS-CoV-2. This article aims to analyze COVID-19 mortality rates and explore their associations with demographic and social indicators in the nine administrative regions of the municipality of Belo Horizonte, the capital of Minas Gerais, which is the second most populous state in Brazil. This study considers and analyzes the spatial-temporal patterns of the COVID-19 deaths reported in the epidemiological bulletins of the Prefeitura de Belo Horizonte $(\mathrm{PBH})(\mathrm{Belo}$ Horizonte city council). The analysis considers the period from Epidemiological Week (EW)EW 23/2020 (31st May 2020) to EW 21/2021 (29th May 2021). The results indicate that the administrative regions had distinct behaviors in relation to COVID-19 and that this performance is predominantly associated with demographic characteristics and social vulnerability.
\end{abstract}

Keywords: Epidemic; Mortality Rates; Demographic and Social Indicators.

\title{
1. INTRODUÇÃO
}

O acesso à informação geográfica se mostrou uma ferramenta imprescindível para ajudar, esclarecer, combater e comunicar dados sobre a dinâmica espaço temporal da pandemia do Coronavírus (COVID-19). Estas ferramentas são particularmente importantes em países com dimensões continentais como o Brasil.

Minas Gerais é uma das 27 unidades federativas do Brasil que foi afetada pela disseminação de COVID-19. Localizado na região sudeste, o estado tem a segunda maior população do país. Sua capital, Belo Horizonte possui 2.523.789 habitantes (PBH, 2018), sendo o quarto PIB per capita (IBGE, 2018) do Brasil, atrás apenas das capitais de São Paulo, Rio de Janeiro e do Distrito Federal. O município está dividido em nove administrações regionais: Barreiro, Centro-Sul, Leste, Nordeste, Noroeste, Norte, Oeste, Pampulha e Venda Nova - divisão que leva em conta a posição geográfica e a história de ocupação. A maior região administrativa, em termos de área, é o Barreiro com 53,6 km² seguida da Pampulha com 51,21 Km² (PBH, 2018).

Belo Horizonte teve o primeiro caso confirmado de COVID-19 em 16 de março de 2020, trata-se de uma mulher de 34 anos de idade. No dia seguinte a Secretária de Estado de Saúde de Minas Gerais (SESMG), confirmou o primeiro caso de transmissão comunitária em Belo Horizonte, um homem de 34 anos de idade. O Decreto Municipal n 17.297, de 17 de março de 2020 declarou emergência em saúde pública, em razão da necessidade de ações para conter a propagação da COVID- 
19, bem como de preservar a saúde da população. Belo Horizonte, foi o quinto munícipio do estado mineiro a confirmar casos da doença e o primeiro a identificar transmissão comunitária (ANDRADE et al., 2020).

Belo Horizonte, foi destaque entre as capitais brasileiras, devido aos resultados satisfatórios no enfrentamento à COVID-19 nos primeiros 80 dias da pandemia. À presença do Sistema Único de Saúde (SUS) e sua organização na capital foram um grande diferencial, uma vez que diversos instrumentos de planejamento e organização da oferta assistencial já estavam consolidados (ANDRADE et al., 2020). As medidas não farmacológicas adotadas no município, em especial, a obrigatoriedade do uso de máscaras e o distanciamento social, além do fechamento de atividades não essenciais e de escolas, ajudaram a controlar a disseminação da COVID-19 no primeiro semestre de 2020 (COUTO et al., 2020; FREITAS et al., 2020; CAIXETA et al., 2020).

No contexto de Minas Gerais, o uso de indicadores, incluindo, demográfico, social, econômico, infraestrutura de saúde, população em risco epidemiológico - permitiu a classificação dos 853 municípios do estado em grupos de 1 (menos vulnerável) a 5 (mais vulnerável). Para critérios demográficos (porcentagem de população vivendo em área urbana; densidade demográfica), a Região Metropolitana de Belo Horizonte se mostrou com alta vulnerabilidade, tendo 42,9\% dos municípios classificados no grupo 5. Para os critérios epidemiológicos (incidência, mortalidade, letalidade de COVID-19 por 1.000 habitantes), a RMBH teve $26,7 \%$ de seus municípios classificados no grupo 5 , o de mais alta vulnerabilidade (CAMPOS et al., 2021).

Desde março de 2020 a Prefeitura de Belo Horizonte (PBH) publica Boletins Epidemiológicos com a evolução da COVID-19 no município. Os registros com inclusão das regionais começaram a ser apresentados nos boletins, de forma mais sistemática, a partir de maio de 2020, com inclusão de óbitos e de casos graves que levaram a internação.

Nesse contexto o artigo tem como principal objetivo analisar as taxas de mortalidade da COVID-19 no município de Belo Horizonte, Minas Gerais com o recorte para suas regionais administrativas. Além disso, pretende-se comparar de forma exploratória, a relação entre as taxas de mortalidade e casos graves de COVID-19 das regiões administrativas da capital mineira com indicadores demográficos e sociais.

\section{MATERIAIS E MÉTODOS}

\section{1. Área de estudo}

A área de pesquisa é o munícipio de Belo Horizonte, capital do estado de Minas Gerais com recorte para suas nove regionais administrativas: Barreiro (BA), Centro-Sul (CS), Leste (LE), Nordeste (NE), Noroeste (NO), Norte (NT), Oeste (OE), Pampulha (PA) e Venda Nova (VN) (Figura 
1). O estudo considera e analisa os padrões espaço-temporais de óbitos por COVID-19 reportados nos boletins epidemiológicos da PBH considerando o período por Semana Epidemiológica (SE), da SE 23/2020 (31 de maio de 2020) até SE 21/2021 (29 de maio de 2021).

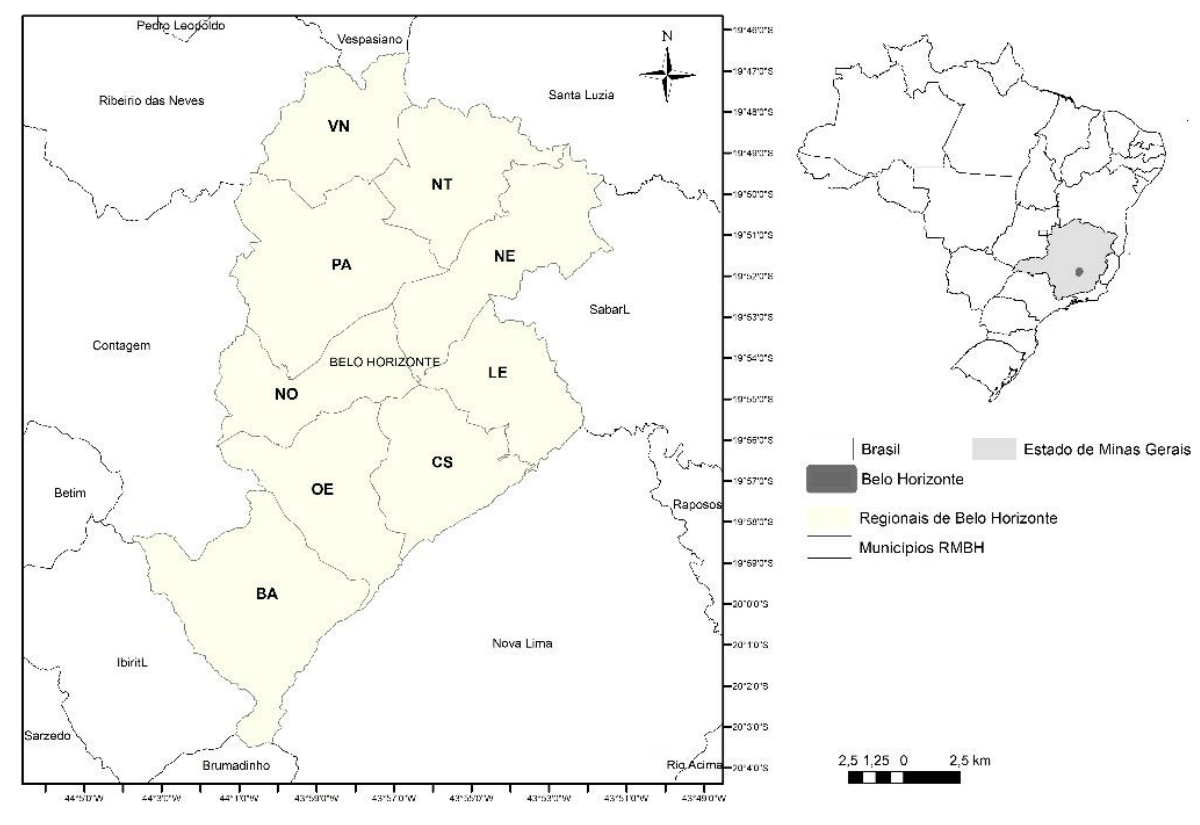

Figura 1 - Mapa de localização do Município de Belo Horizonte.

Fonte: Dados da pesquisa.

\subsection{Taxas de Mortalidade, Casos Graves e Análises Espaciais}

Considerando o período analisado os dados foram agregados, de forma acumulada, por períodos de quatro Semanas Epidemiológicas (SEs). Essa escolha foi baseada na proposta de CouraVital et al., (2021) que realizaram análise similar para todos os municípios do estado de Minas Gerais, entretanto, a cobertura dos dados foi apenas parcial (primeiro semestre de 2020). O recorte temporal do período de SE 23/2020 (31 de maio de 2020) até SE 21/2021 (29 de maio de 2021), foi escolhido tanto pelo fato das informações sistemáticas das regionais estarem disponíveis apenas a partir do mês de maio de 2020, como pelo fato desse período cobrir de forma satisfatória as duas primeiras ondas pandêmicas registradas em Minas Gerais. A $1^{\text {a }}$ onda ocorreu entre 08 de março de 2020 e 31 de outubro de 2020, com pico de óbitos entre 16 e 22 de agosto de 2020; e a $2^{\mathrm{a}}$ onda foi registrada entre $1^{\circ}$ de novembro de 2020 e $1^{\circ}$ de maio de 2020, com pico de óbitos entre 11 e 17 de abril de 2021 (CERQUEIRA et al., 2021 - no prelo).

Os dados populacionais por regional são aqueles apresentados no ano de 2010 pelo IBGE, dados gerados a partir do último Censo. Para calcular as taxas de incidência por 100 mil habitantes, cada caso foi agregado pela regional de residência. $\mathrm{O}$ banco de dados com os óbitos por regional foi 
feito no Microsoft Excel e unido ao banco de dados espacial no software ArcGis 10.5 para elaboração dos mapas e análise espacial. Para a análise foram considerados os dados acumulados.

\subsection{Caracterização das Regionais por Indicadores Demográficos e Sociais}

Para auxiliar no debate sobre a relação entre os óbitos por COVID-19 e as características das regionais, alguns dados demográficos e sociais foram representados em mapas: o Índice de Vulnerabilidade à Saúde (IVS); a ocorrência de Vilas e Favelas; assim como a Porcentagem da população acima de 60 anos (faixa etária onde se concentra a maioria dos óbitos) usando como referência os setores censitários do IBGE de 2010 e os dados espaciais abertos no portal de geoprocessamento da PBH.

\section{RESULTADOS}

\subsection{Evolução Espacial dos Óbitos por Covid-19}

A evolução de óbitos por COVID-19, considerando as regionais de Belo Horizonte, é apresentada na Figura 2.
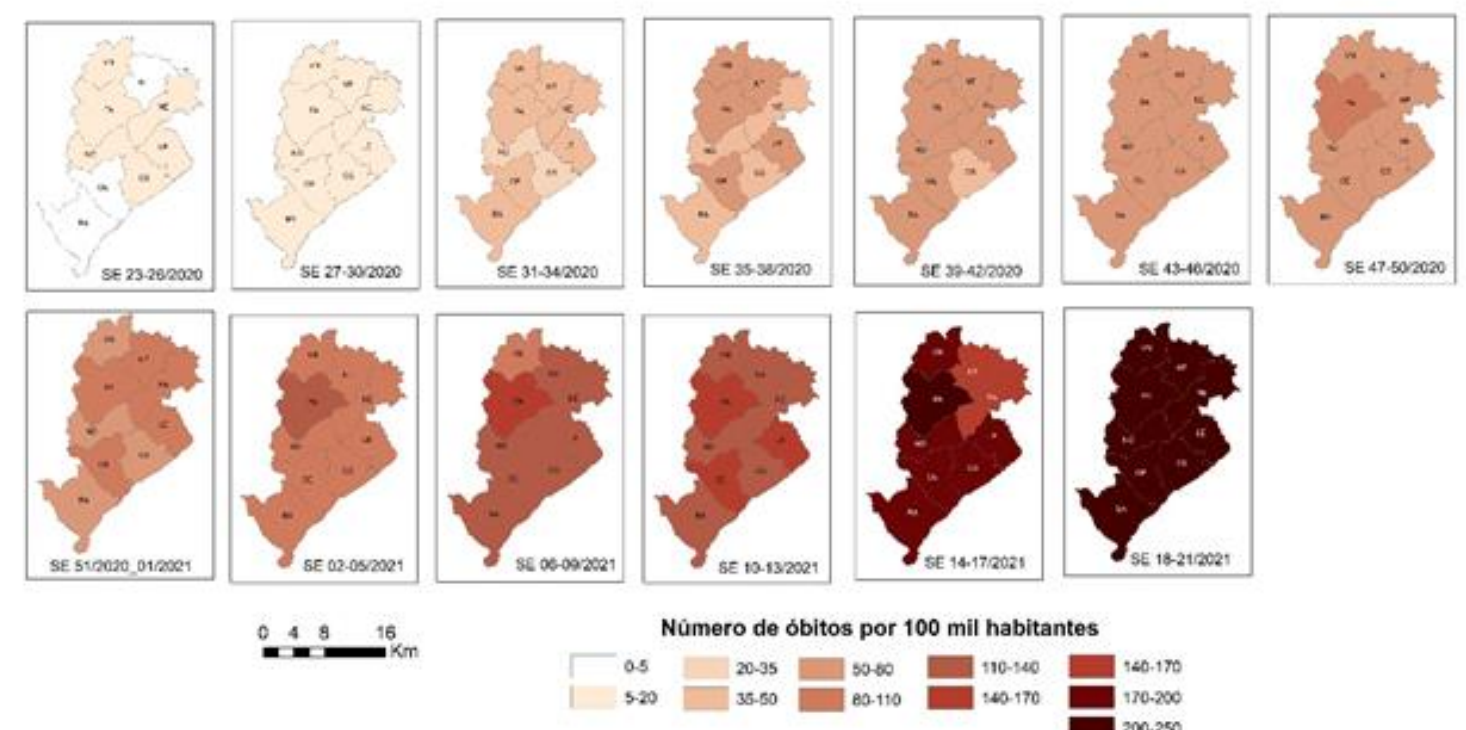

Figura 2 - Evolução dos óbitos acumulados considerando as regionais do município de Belo Horizonte. A primeira onda finaliza em 31/10/2020 (SEs 43-44/2020). A análise considera a segunda onda com início em 01/11/2020 (SEs 4546/2020) até o 29/05/2021 (SEs 18-20/2021).

Fonte: os autores (2021).

O primeiro óbito em Belo Horizonte por COVID-19 foi registrado em 19 de março de 2020. Nas quatro primeiras semanas analisadas (SE 23-26/2020) a maioria das regionais apresentam de 5 a 20 óbitos por 100 mil/habitantes, com exceção das regionais Norte, Oeste e Barreiro com 0 a 5 óbitos por $100 \mathrm{mil} /$ habitantes. Sendo a regional Leste aquela com maior registro $(6,01)$ seguida das regionais 
Pampulha $(5,87)$ e Nordeste $(5,83)$. Nas quatro semanas seguintes (SE 27-30/2020) todas as regionais já registram entre 5 e 20 óbitos por 100 mil habitantes. Nas SE 31-34/2020 e SE 35-38/2020 as regionais com menor número de óbitos são as Nordeste $(57,71)$, Noroeste $(53,41)$, Centro-Sul $(47,74)$ e Barreiro $(53,79)$. Nas quatro semanas seguintes (SE 39-42/2020) a única regional que mantém taxas mais baixas no intervalo considerando, quando comparada as demais é a Centro-Sul. A partir da SE 47-50/2020, a regional Pampulha aparece sempre com a primeira entre as regionais a atingir as maiores taxas de mortalidade. Esse período se dá logo após o fim da primeira onda em 31 de outubro de 2020 (SE 43/44/2020) e início da segunda onda (CERQUEIRA et al., 2021 - no prelo).

As regionais com maior número de óbitos acumulados por 100.000 habitantes, ao fim da primeira onda são: Pampulha $(79,01)$; Norte $(73,25)$; Venda Nova $(69,79)$ e Leste $(69,41)$. Entre as regionais com menor número de óbitos acumulados por 100.000 habitantes estão: Centro-Sul (54, 35); Barreiro $(59,45)$ e Noroeste $(60,96)$.

A regional Pampulha é a primeira a atingir o intervalo entre 80-110 óbitos (SEs 47-50/2020), seguida das regionais Norte, Noroeste, Leste e Oeste nas quatro semanas seguintes (SEs 51/202001/2021). As regionais Pampulha, Leste e Oeste continuam entre as primeiras a marcarem o aumento no intervalo de óbitos nas semanas epidemiológicas subsequentes (até SEs 10-13/2021). Na SEs 1417/2021 a regional Pampulha apresenta registro no intervalo de 200 a 250 óbitos; as regionais Norte e Noroeste seguem apresentando menor registro de óbitos (140-170) e todas as outras regionais já se encontram no intervalo de 170-200 óbitos por 100.000 habitantes.

\subsection{Situação das Regionais em Relação a Covid-19}

A tabela 1 apresenta os casos graves e óbitos acumulados por 100.000 habitantes considerando o período analisado para: a $1^{\text {a }}$ onda (SE 23/2020 a SE 44/2020 - com dados acumulados do início da pandemia); a $2^{\text {a }}$ onda (SE 45/2020 a SE 21/2021); e para todo o período (SE 23/2020 (já acumulado com o início da pandemia) até SE 21/2021). Para facilitar a análise os dados das regionais foram agrupados em três cores diferentes: vermelho (para as três piores regionais em relação ao dado analisado no período indicado); amarelo (para as três regionais em situação intermediária); verde (para as três regionais em situação melhor).

Ao fim da primeira onda as regionais com maior número de óbitos foram: PA $(74,74)$; NT $(68,09)$ e LE $(66,99)$. As regionais com número de óbitos intermediários são VN $(66,75)$; OE $(62,56)$ e NE $(61,49)$. As regionais com menor número de óbitos ao final da primeira onda são: CS $(52,12)$; BA $(57,33)$ e NO $(58,85)$. Ao final da segunda onda a única regional que permanece na mesma categoria (entre as três com maior taxa de mortalidade) é a PA (174,04). As regionais CS e BA pioram seu desempenho em relação a primeira onda e passam a figurar entre as três mais letais, com 
respectivamente, 164,53 e 155,02 óbitos por 100.000 mil habitantes. A regional NO permanece, na segunda onda entre as três menos letais (comportamento já observado ao final da primeira onda), com 142, 14 óbitos.

Tabela 1: Casos graves e óbitos acumulados nas regionais de Belo Horizonte. Os dados são agrupados em três cores: verde (as três regionais em melhor situação); amarelo (as três regionais em situação intermediária); vermelho (as três regionais em pior situação).

\begin{tabular}{|c|c|c|c|c|c|c|}
\hline \multirow{2}{*}{ Regional } & \multicolumn{2}{|c|}{$\begin{array}{c}\text { SE 23/2020 a SE 44/2020 } \\
\left(1^{\text {a }} \text { onda }\right)\end{array}$} & $\begin{array}{r}\text { SE 45/2020 a SE 21/2021 } \\
\left(2^{\mathrm{a}} \text { onda) }\right.\end{array}$ & \multicolumn{2}{c|}{$\begin{array}{r}\text { SE 23/2020 a SE 21/2021 } \\
\text { (acumulado) }\end{array}$} \\
\cline { 2 - 7 } & Casos SRAG & Óbitos & Casos SRAG & Óbitos & Casos SRAG & Óbitos \\
\hline BA & 266,50 & 57,33 & 610,15 & 155,02 & 876,65 & 212,35 \\
\hline CS & 223,66 & 52,15 & 564,11 & 164,53 & 797,77 & 216,68 \\
\hline LE & 315,72 & 66,99 & 688,00 & 151,24 & $1,003,72$ & 218,23 \\
\hline NE & 268,63 & 61,49 & 668,13 & 152,86 & 936,76 & 214,35 \\
\hline NO & 219,10 & 58,85 & 542,01 & 142,14 & 761,11 & 200,99 \\
\hline NT & 276,59 & 68,09 & 583,23 & 138,53 & 859,82 & 206,62 \\
\hline OE & 307,91 & 62,56 & 471,13 & 146,09 & 779,04 & 208,65 \\
\hline PA & 300,03 & 74,74 & 643,84 & 174,04 & 943,87 & 248,78 \\
\hline VN & 268,13 & 66,75 & 492,79 & 134,26 & 760,92 & 201,01 \\
\hline
\end{tabular}

Fonte: os autores (2021).

\subsection{Indicadores Sociais e Demográficos das Regionais}

A tabela 2 apresenta os casos graves e óbitos acumulados por 100.000 habitantes considerando o período analisado (SE 23/2020 (já acumulado com o início da pandemia) até SE 21/2021) e inclui a densidade demográfica e a porcentagem da população acima de 60 anos e entre 40 e 59 anos. Essas são as duas faixas etárias para as quais a COVID-19 apresentou maior índice de mortalidade no período analisado.

Tabela 2: Casos graves e óbitos acumulados e sua associação com dados demográficos (densidade demográfica e porcentagem da população acima de 60 anos e entre 40 e 59 anos).

\begin{tabular}{|c|c|c|c|c|c|c|c|c|c|c|c|}
\hline \multirow[t]{2}{*}{ Regional } & \multirow[t]{2}{*}{$\begin{array}{l}\text { População } \\
\text { (2010) }\end{array}$} & \multirow[t]{2}{*}{$\begin{array}{l}\text { Area } \\
\left(\mathrm{km}^{2}\right)\end{array}$} & \multirow[t]{2}{*}{$\begin{array}{c}\text { Densidade demografica } \\
\text { (hab/ } \mathrm{km}^{2} \text { ) }\end{array}$} & \multicolumn{2}{|c|}{ Faixa etária (\%) } & \multicolumn{2}{|c|}{$\begin{array}{c}\text { SE } 23 / 2020 \text { a SE } \\
44 / 2020 \\
\left(1^{*} \text { onda }\right)\end{array}$} & \multicolumn{2}{|c|}{$\begin{array}{c}\text { SE } 45 / 2020 \text { a SE } \\
21 / 2021 \\
\left(2^{*} \text { onda }\right)\end{array}$} & \multicolumn{2}{|c|}{$\begin{array}{c}\text { SE } 23 / 2020 \text { a SE } \\
21 / 2021\end{array}$} \\
\hline & & & & $\geq 60$ & $\geq 40<60$ & $\begin{array}{l}\text { Casos } \\
\text { SRAG }\end{array}$ & Óbitos & $\begin{array}{l}\text { Casos } \\
\text { SRAG }\end{array}$ & Óbitos & $\begin{array}{l}\text { Casos } \\
\text { SRAG }\end{array}$ & Óbitos \\
\hline $\mathrm{BA}$ & 282552 & 53,60 & 5271.49 & 9,73 & 23,91 & 266.50 & 57,33 & 610,15 & 155,02 & 876,65 & 212,35 \\
\hline $\mathrm{CS}$ & 272285 & 31,85 & 8548,98 & 18.69 & 26,47 & 223,66 & 52,15 & 564,11 & 16453 & 797.77 & 21606 \\
\hline LE & 249273 & 27,98 & 8000,97 & 14.20 & 26.02 & $3 \leq 72$ & 66.69 & $68 \times 600$ & 151,24 & $1,003,23$ & $21 \times 22$ \\
\hline $\mathrm{NE}$ & 291110 & 39,46 & 7377,34 & 11,80 & 25,60 & 268,63 & 61.49 & $60 \times 13$ & 152,86 & 93676 & 214,35 \\
\hline No & $231 \times 2$ & 30,17 & $109 \times 3,16$ & $1+39$ & 2652 & 219.10 & 58,85 & $\$ 42,01$ & 142,14 & 761,11 & 200,99 \\
\hline NT & 212953 & 32,67 & 6518,30 & 9.94 & 23,84 & 276,59 & $\sin (0)$ & 583,23 & 138.53 & 859,82 & 206.62 \\
\hline $\mathrm{OE}$ & 286118 & 36,06 & 7934,50 & 12,36 & 25,54 & 307.91 & 62,56 & 471,13 & 146,09 & 779,04 & 208,65 \\
\hline $\mathrm{PA}$ & 187315 & 5121 & 3657,78 & 10.93 & 25,65 & 30003 & 74,74 & $64 \times \times 4$ & 174,04 & $041 \times 5$ & 24878 \\
\hline $\mathrm{VN}$ & 262183 & 29,27 & $x_{0.57}, 40$ & 9.94 & 23.77 & 268,13 & 66,75 & 492.79 & 134.26 & 760,92 & 201,01 \\
\hline
\end{tabular}

Fonte: os autores (2021). 
A figura 3 apresenta a distribuição de bairros, favelas e vilas; o Índice de Vulnerabilidade a Saúde (IVS) para todas as regionais (PBH, 2012); bem como a distribuição etária para as regionais considerando o setor censitário e as classes de porcentagem da população em cada setor acima de 60 anos.

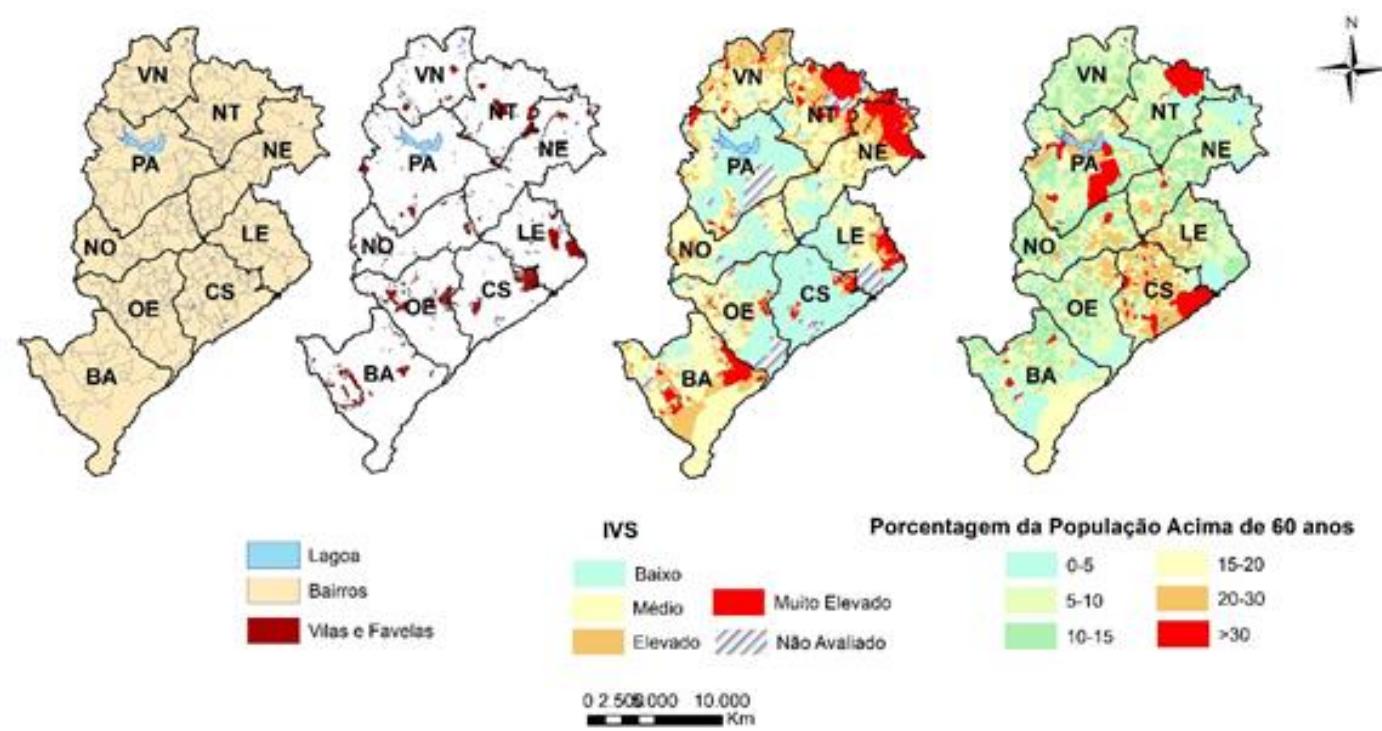

Figura 3 - Distribuição de bairros (a); vilas e favelas (b); IVS (c) e classes de porcentagem da população acima de 60 anos (d) das regionais de Belo Horizonte.

Fonte: PBH: 2012.

As quatro regionais com maiores densidades demográficas (acima de 8.000,0 hab $/ \mathrm{km}^{2}$ ): Noroeste $(10.983,16)$; Venda Nova $(8.957,40)$; Leste $(8.908,97)$ e Centro Sul $(8.548,98)$ - tiveram um comportamento diferenciado em relação a evolução de COVID-19 no período analisado.

A regional Noroeste, embora com mais alta densidade demográfica é uma das que apresentou menor número de casos graves e internações por COVID-19 e a que apresentou menor número de óbitos. O mesmo comportamento teve a regional Venda Nova que apresenta alta densidade demográfica, mas um número reduzido de internações e mortes por COVID-19. As regionais CentroSul e Leste estão entre as três com maior número de óbitos e a regional Leste está entre as três com maior número de internações. A regional Pampulha, embora tenha a menor densidade demográfica entre as regionais, figura entre as três com maior número de óbitos e internações.

O Índice de Vulnerabilidade da Saúde (IVS) (Figura 3 (c)), é calculado para cada conjunto de pessoas residentes em uma determinada área geográfica contínua (setor censitário do IBGE), classificada em 4 categorias: médio risco, baixo risco, risco elevado e muito elevado (CLARET, 2017). Para o cálculo são considerados indicadores sanitários e socioeconômicos. A dimensão sanitária tem como indicadores: percentual de domicílios particulares permanentes com abastecimento de água inadequado ou ausente; percentual de domicílios particulares permanentes 
com esgotamento sanitário inadequado ou ausente e percentual de domicílios particulares permanentes com destino de lixo de forma inadequada ou ausente. A dimensão socioeconômica considera os seguintes indicadores: percentual de pessoas analfabetas; percentual de domicílios particulares com rendimento per capita até meio salário-mínimo; rendimento nominal mensal médio das pessoas responsáveis (invertido), e; percentual de pessoas de raça/cor preta, parda e indígena (CLARET, 2017).

Ao se analisar a ocorrência de vilas e favelas, bem como o Índice de Vulnerabilidade à Saúde (Figura 3 (c)), a regional Noroeste praticamente não apresenta áreas classificadas como muito alto IVS, sendo que a maior parte da regional recebe classificação de baixo a médio IVS. A ocorrência de vilas e favelas também é relativamente pequena, quando comparada as demais regionais. Essa regional figura entre as três com menor taxa de mortalidade e internações por COVID-19 embora seja a de maior densidade demográfica e esteja entre as três com maior porcentagem de população com faixa etária mais atingida (acima de 60 e entre 40 e 60 anos).

A regional Leste, que figura entre as mais afetadas em termos de internação e óbitos por COVID-19, apresenta maior concentração de vilas e favelas, em especial na divisa com a regional Centro-Sul (também bastante afetada pela pandemia) e na sua porção mais a leste. Essas áreas correspondem aquelas classificadas como de muito elevado IVS. Esses dados, associados a alta densidade demográfica, podem justificar o desempenho em relação a COVID-19 em comparação com as demais regionais. O mesmo raciocínio vale para a regional CS.

As regionais NT, NE e BA também apresentam áreas classificadas como de muito elevado IVS, no entanto figuram entre as quatro com menor densidade demográfica juntamente com a regional PA.

Considerando os boletins epidemiológicos da $\mathrm{PBH}$ para as duas primeiras ondas registradas em Minas Gerais ( $1^{\mathrm{a}}$ onda 31/10/2020 - Boletim 136/2020) e $\left(2^{\mathrm{a}}\right.$ onda 29/05/2021 - Boletim 281/20210), o percentual de óbitos por faixa etária é apresentado na Tabela 3.

Tabela 3: Porcentagem de óbitos por faixa etária segundo Boletins Epidemiológico dos períodos analisados.

\begin{tabular}{|l|c|c|}
\hline Faixa & $\begin{array}{c}\text { Boletim 136/2020 } \\
\left(\mathbf{0 3 / 1 1 / 2 0 2 0 )}-\mathbf{1}^{\mathbf{a}}\right. \\
\text { onda }\end{array}$ & $\begin{array}{c}\text { Boletim 281/2021 } \\
\left(\mathbf{0 1 / 0 6 / 2 0 2 1 )}-\mathbf{2}^{\text {a }}\right. \\
\text { onda }\end{array}$ \\
\hline $\mathbf{0 - 1 9}$ & $0,1 \%$ & $0,1 \%$ \\
\hline $\mathbf{2 0 - 3 9}$ & $2,2 \%$ & $2,8 \%$ \\
\hline $\mathbf{4 0 - 5 9}$ & $15,1 \%$ & $16,3 \%$ \\
\hline$>\mathbf{6 0}$ & $82,6 \%$ & $80,7 \%$ \\
\hline
\end{tabular}

Fonte: PBH, 2012. 
Em relação ao perfil etário, a figura 03 (d) mostra a distribuição da população acima de 60 anos (que concentra o maior número de óbitos pela COVID-19) nas regionais. Os dados consideram cada conjunto de pessoas residentes em uma determinada área geográfica contínua (setor censitário do IBGE), e referem-se ao último Censo Demográfico do IGBE de 2010. A porcentagem da população por regional nas diferentes faixas etárias é apresentada também na Tabela 04.

Tabela 4: Porcentagem da população nas diferentes faixas etárias considerando os dados demográficos do Censo de 2010.

\begin{tabular}{|l|c|c|c|c|c|c|c|c|c|}
\hline $\begin{array}{c}\text { Faixa } \\
\text { Etária }\end{array}$ & BA & CS & LE & NE & NO & NT & OE & PA & VN \\
\hline $\mathbf{0 - 1 9}$ & $29 \%$ & $20,6 \%$ & $24,3 \%$ & $26,2 \%$ & $23,1 \%$ & $29,1 \%$ & $24,5 \%$ & $25,2 \%$ & $28,3 \%$ \\
\hline $\mathbf{2 0 - 3 9}$ & $35,9 \%$ & $34,9 \%$ & $34,1 \%$ & $35,2 \%$ & $34,2 \%$ & $35,9 \%$ & $35,8 \%$ & $36,2 \%$ & $36,5 \%$ \\
\hline $\mathbf{4 0 - 5 9}$ & $23,8 \%$ & $26,1 \%$ & $26,1 \%$ & $25,4 \%$ & $26,7 \%$ & $23,9 \%$ & $25,6 \%$ & $25,3 \%$ & $23,9 \%$ \\
\hline$>\mathbf{6 0}$ & $9,8 \%$ & $17,5 \%$ & $14,5 \%$ & $11,8 \%$ & $14,7 \%$ & $9,8 \%$ & $12,4 \%$ & $11,5 \%$ & $10,0 \%$ \\
\hline$>\mathbf{4 0}$ & $33,6 \%$ & $43,6 \%$ & $40,6 \%$ & $37,2 \%$ & $41,4 \%$ & $33,7 \%$ & $38,0 \%$ & $36,8 \%$ & $33,9 \%$ \\
\hline
\end{tabular}

Fonte: Censo (2010).

Até o final da primeira onda (31/10/2020) 82,6\% dos óbitos registrados por COVID-19 foram na faixa etária acima dos 60 anos. Entre a primeira e a segunda onda os óbitos passam a atingir também a população abaixo de 60 anos e coincide com o início da vacinação para a população idosa de Belo Horizonte, em 18 de janeiro de 2020 - isso tem resultado na porcentagem de óbitos que, acumulando a primeira e a segunda onda, passa para 80,7\% acima de 60 anos. Segundo dados do censo do IBGE de 2010 as regionais com maior população nessa faixa etária são: Centro-Sul (17,5\%); Noroeste $(14,7 \%)$ e Leste (14,5\%). As regionais com menor população proporcional nesse faixa etária são Barreiro e Norte $(9,8 \%)$ e Venda Nova (10\%). As regionais CS e LE estão entre as mais afetadas pela pandemia de COVID-19.

A regional Pampulha pode ser considerada um caso emblemático que merece um estudo mais aprofundado. Essa regional, diferente das demais que apresentaram maior vulnerabilidade para COVID-19, apresenta poucas áreas classificadas como de elevado IVS, poucas vilas e favelas, baixa densidade demográfica, e porcentagem da população na faixa etária mais atingida em posição intermediária quando comparada as demais regionais.

No caso das demais regionais, o comportamento em relação a COVID-19 (taxa de mortalidade e número de internações por casos graves) é o resultado combinado de: alta densidade demográfica; maior porcentagem da população nas faixas etárias mais atingidas; maior número de áreas classificadas como de muito elevado IVS. 


\section{CONSIDERAÇÕES FINAIS}

Os resultados deste trabalho contribuem para uma maior compreensão da dinâmica espaço temporal da epidemia COVID-19 em Belo Horizonte distinguindo suas regionais administrativas que apresentam características distintas considerando: densidade demográfica; percentagem da população com idade de maior risco para COVID-19; Índice de Vulnerabilidade Social; presença de vilas e favelas. A espacialização espaço temporal dos óbitos ao longo das semanas epidemiológicas SE 23/2020 até SE 21/2021, mostra que as regionais que começaram a segunda onda em situação mais críticas se mantiveram assim até o período analisado. O estudo baseado em análise espaço-temporal está sujeito a limitações, mas pode ser usado para compreender a dinâmica de COVID-19 em Belo Horizonte considerando suas diferentes regionais administrativas.

A maior limitação do estudo se deve a oferta de dados secundários, em especial aqueles produzidos pelo IBGE que teve seu último censo publicado em 2010. Dados demográficos mais recentes podem ter um forte impacto nos resultados apresentados e poderiam, por exemplo, justificar o desempenho da regional Pampulha que não apresenta nenhum dado social ou demográfico (considerando dados de 2010) que expliquem sua situação quando comparada as demais regionais. O comportamento dessa regional poderia ser melhor compreendido frente a dados demográficos mais atuais. Também importante seria o monitoramento e disponibilização de outras variáveis acerca do comportamento da população em relação ao cumprimento do distanciamento social em período de pandemia. Por exemplo, o não cumprimento do distanciamento poderia explicar a situação da regional Pampulha.

Após analisar cuidadosamente tanto as bases de dados disponíveis como a literatura afins, pudemos explorar os aspectos relacionados entre essa nova doença e as diferentes características das regionais de Belo Horizonte. Em caso de pandemia o acompanhamento e espacialização dos dados em mapas pode auxiliar o poder público no enfrentamento da doença, inclusive direcionando esforços de fiscalização em regiões mais afetadas que podem ser consideradas prioritárias.

\section{AGRADECIMENTOS}

UAR e SRC agradecem ao CNP pela Bolsa de Produtividade em Pesquisa e NC pela Bolsa de Iniciação Científica. EOCJ e MLC agradecem a CAPES pela Bolsa de Pós-Doutorado e pela Bolsa de Doutorado. Os autores agradem ao apoio da CAPES no financiamento do Projeto de Pesquisa aprovado no Edital de Seleção Emergencial "Prevenção e combate a surtos, endemias, epidemias e pandemias" - Edital número 9/2020. 


\section{REFERÊNCIAS}

ANDRADE, M. V.; NORONHA, K.; TURRA, C. M.; GUEDES, G.; CIMINI, F.; RIBEIRO, L. C.; BERNARDES, A. T.; DOMINGUES, E.; RIBEIRO, M. M.; BOTEGA, L. A.; CARVALHO, L. R.; NOGUEIRA, D.; CALAZANS, J. A.; JULIÃO, N. A.; SILVA, A. S.; ANDRADE, V.; LIMA, V. H.; ANDRADE, J. P.; FERREIRA, M. F.; SANTOS, R. O.; SILVA, J. A. Os primeiros 80 dias da pandemia da COVID-19 em Belo Horizonte: da contenção à flexibilização. Nova Economia, v. 30, n. 2, p. 701-737, 2020.

CAIXETA, L. P.; SILVA, T. R.; ANTUNES, D. E. Analysis of COVID-19 pandemic trends and its impact on the health system of the main urban centers of Minas Gerais, Brazil. International Journal for Innovation Education and Research, v.8, n.11, p. 621-633, 2020.

CAMPOS, I. S.; ARATANI, V. F.; CABRAL, K. B.; LIMONGI, J. E.; OLIVEIRA, S. V. A vulnerability analysis for the management of and response to the COVID-19 epidemic in the second most populous state in Brazil. Frontiers in Public Health, v. 9, p. 1-37, 2021.

CERQUEIRA, E. D. V.; CAMPOS JÚNIOR, E. O.; FERREIRA, Á. G. A.; DIOGO, A. M.; FONSECA, B. M.; NASCIMENTO, D. C. M.; CORTEZ, M. L.; RIBEIRO, S. M. C.; RUCHKYS, Ú. A.; COELHO, V. B. N. A COVID-19 em Minas Gerais: uma análise sóciodemográfica das duas ondas da pandemia. In: Panorama da COVID-19 no Brasil. Curitiba: Editora CRV, 2021.

CLARET, T. A. M. A política da promoção da saúde de Belo Horizonte. 2017. 103 f. Dissertação (Mestrado em Promoção de Saúde e Prevenção da Violência) - Faculdade de Medicina, Universidade Federal de Minas Gerais, Belo Horizonte, 2017.

COURA-VITAL, W.; CARDOSO, D. T.; KER, F. T. O.; MAGALHÃES, F. C.; BEZERRA, J. M. T.; VIEGAS, A. M.; MORAIS, M. H. F.; BASTOS, L. S.; REIS, I. A.; CARNEIRO, M.; BARBOSA, D. S. Spatiotemporal dynamics and risk estimates of COVID-19 epidemic in Minas Gerais State: analysis of an expanding process. Revista do Instituto de Medicina Tropical de São Paulo. v. 63, p. 1-12, 2021.

COUTO, B.; ALVIM, A. L.; JUNIOR, J. C.; STARLING, C. Cenários atuais de transmissão da COVID-19 em Belo Horizonte/MG e Itabuna/BA: análise das primeiras 4 semanas da pandemia. Journal of Infect Control, v. 9, n. 2, p. 51-57, 2020.

FREITAS, E. S. M.; NUNES, M. S.; SILVA, P. E. A. B. A materialidade geohistórica e epidemiológica da COVID-19: reflexões e análises no contexto da Região Metropolitana de Belo Horizonte. Boletim Goiano de Geografia, v. 40, p. 1-27, 2020.

IBGE - INSTITUTO BRASILEIRO DE GEOGRAFIA E ESTATÍSTICA. Projeções da população: Brasil e unidades da Federação. 2. ed. Rio de Janeiro: IBGE, 2018. Disponível em: https://goo.gl/y1UwJc. Acesso em: 5 jul. 2021.

IBGE - INSTITUTO BRASILEIRO DE GEOGRAFIA E ESTATÍSTICA. Censo Brasileiro de 2010. Rio de Janeiro: IBGE, 2012. Disponível em: https: www.ibge.gov.br/estatisticas/downloadsestatisticas.html. Acesso em: 20 mai. 2021.

PBH - PREFEITURA DE BELO HORIZONTE. Dados abertos da PBH, 2020, 2021. Disponível em: https://prefeitura.pbh.gov.br/saude/coronavirus. Acesso em: 23 mai. 2021. 
PBH - PREFEITURA DE BELO HORIZONTE. Dados abertos da PBH, 2012. Disponível em: https://prefeitura.pbh.gov.br/bhgeo/geoprocessamento-pbh. Acesso em: 23 mai. 2021.

Trabalho enviado em 21/05/2021

Trabalho aceito em 04/08/2021 